\title{
Evaluación de salchichas elaboradas con carne roja de atún
}

\author{
Evaluation of red meat sausage made with tunahead
}

\author{
Avaliação de salsichas feitas com atum carne vermelha
}

Clemente Granados-Conde ${ }^{1 *}$, Luis E. Guzmán-Carrillo ${ }^{2 *}$, Diofanor Acevedo-Correa ${ }^{3 *}$

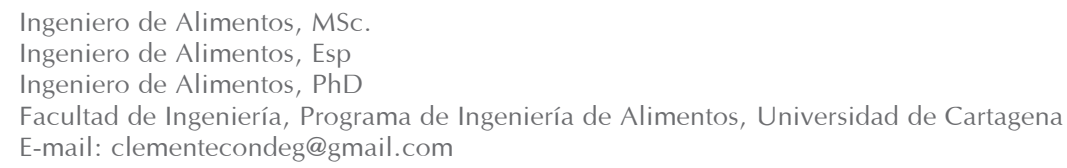

\section{Resumen}

Se elaboró salchichas con carne roja de atún y se determinó sus características fisicoquímicas, microbiológicas, sensoriales y de textura. La composición proximal se determinó según la Asociación Oficial de Química Analítica. Las características microbiológicas se determinó según las Normas Técnicas Colombianas. La aceptabilidad fue evaluada con panelistas no entrenados, utilizando una escala hedónica para sabor, color, olor y textura. Los contenidos proximales fueron: humedad $(67,25)$, grasa $(8,2 \%)$, proteínas $(17,3 \%)$ y cenizas $(1,95 \%)$. Las salchichas presentaron buena calidad microbiológica y sensorial.

Palabras Claves: atún, salchichas, textura, sensorial, calidad.

\begin{abstract}
Sausage was prepared with red meat of tuna and it's physicochemical, microbiological and sensorial characteristics, and texture were determined. The proximate composition was determined according to the Analytical Chemistry Official Association. Microbiological characteristics were determined according to Colombian Technical Standards. Acceptability was evaluated with untrained panelists using a hedonic scale for taste, color, smell and texture. Proximal contents were: moisture (67.25), fat (82\%), protein (17.3\%) and ash (1.95\%). The sausages had good microbiological and sensorial quality.
\end{abstract}

Keywords: tuna, sausage, texture, sensory, quality.

\section{Resumo}

Foram preparadas salchichas com carne vermelha de atum e determinadas as suas propriedades físico-químicas, microbiológicas, características sensoriais e de textura. A composição centesimal foi determinada de acordo com a Association of Official Analytical Chemistry; as características microbiológicas de acordo com as Normas Técnicas colombianas e a avaliada a aceitabilidade com provadores não treinados utilizando uma escala hedônica para sabor, cor, cheiro e textura. Os conteúdos proximais foram humidade $(67,25)$, gordura $(8,2 \%)$, proteína $(17,3 \%)$ e cinzas $(1,95 \%)$. As salsichas apresentaram boa qualidade microbiológica e sensorial.

Palavras-chave: atum, salsicha, textura, qualidade sensorial. 


\section{Introducción}

Los atunes son peces de la familia Scombridae del género Thunnus. Son de gran aceptación ya que tienen un alto contenido de compuestos nutritivos como proteínas de alto valor biológico y fácil digestibilidad, vitaminas liposolubles e hidrosolubles, minerales como yodo, fósforo, hierro, magnesio, potasio y ácidos grasos poliinsaturados, Omega-3 y Omega-6 (Noguez et al., 2007). Las excelentes propiedades del atún y su importante volumen de captura lo hacen una de las especies marinas de mayor potencial para el desarrollo y manufactura de gran variedad de productos. Entre ellos, diversos tipos de embutidos y enlatados (García 2005; Izquierdo et al 2007).

En el proceso de producción de atún enlatado se generan subproductos como carne de cabeza, de cara y residuos de las salas de proceso, desde la captura hasta el despacho final. Esta carne representa entre un $20-30 \%$ del peso. Por ello, una alternativa para aumentar los rendimientos es aprovecharla elaborando productos como el grated, o "atún rallado", que es una mezcla de partículas reducidas a dimensiones uniformes, mediante molinos adecuados (Izquierdo et al 2007).

La salchicha es una de las formas más antiguas de procesar alimentos, y ha sobresalido por sus características nutricionales, sensoriales y funcionales respecto a otros productos cárnicos (Izquierdo et al, 2007; Tinedo, 1998). Algunas investigaciones evidencian la ventaja de utilizar diversos tipos de carnes en su elaboración con el fin de diversificar la presentación al consumidor, sobre todo al infantil, que representa un sector amplio (García et al 2005; Granados et al., 2012). Las salchichas se clasifican dentro del grupo de los embutidos escaldados, compuestos por una mezcla finamente picada de tejido muscular (carne), tejido graso y agua, a la que se le añade sal y especias para la formación del color, sabor y, en parte, para su estabilización. Esa mezcla es empacada en una tripa, la cual es sellada y luego hervida, o sometida al vapor (Hleap y Molina,2008). En la elaboración de salchichas con carne de pescado se han presentado dificultades, entre ellas la búsqueda de la proporción adecuada de los ingredientes, con el fin de obtener una apariencia y un sabor aceptables por hábitos alimenticios arraigados en diferentes regiones.

Por lo anterior, el objetivo de este trabajo fue elaborar salchichas con la carne roja de la cabeza del atún y evaluar sus características fisicoquímicas, microbiológicas, sensoriales y de textura.

\section{Materiales y métodos}

\section{Obtención de la materia prima}

La carne roja de la cabeza del atún se adquirió por medio de una empresa procesadora de atún enlatado de la ciudad de Cartagena-Colombia y se mantuvo empacada en bolsas plásticas a $-18^{\circ} \mathrm{C}$ hasta su utilización. La grasa de cerdo se adquirió en un supermercado de la ciudad. Los demás ingredientes que hacen parte de la mezcla se adquirieron en un almacén especializado en insumos para embutidos cárnicos.

La carne roja de atún fue descongelada y sometida a un lavado en una solución de cloruro de sodio al $0.9 \%$ para obtener una carne clara, sin sabor y sin su olor característico, ya que éste le imprime un sabor penetrante aún después del escaldado. El lavado de carne de atún se basa en el proceso de elaboración de surimi. El mismo empieza con un picado fino de la carne a tratar. Después esta carne es pesada con el fin de obtener la relación de agua/carne. Se utilizó la relación de 6 partes de agua por cada parte de carne de atún. La carne a tratar y la cantidad de agua necesaria para el proceso se colocan en un recipiente. Es importante señalar que el agua debe de estar entre 5 y $10^{\circ} \mathrm{C}$ con el fin de mantener la calidad del pescado. Luego se procede a agitar el contenido del recipiente por 20 minutos. Después este contenido se deja en reposo por 20 minutos más y se elimina el agua del lavado. Este proceso se repite tres veces. Luego de cada periodo de reposo se debe eliminar el agua y se agrega agua limpia. Como paso final, se escurre el agua utilizada tratando de dejar la carne sin restos de agua, con la ayuda de un tamiz.

Para el proceso de elaboración de las salchichas se siguió el procedimiento empleado por Hleap y Molina (2008). La carne roja de la cabeza de atún, la grasa de cerdo, la mitad del hielo y los demás ingredientes se colocaron en un cutter Mainca, modelo CM14, dotado de termómetro en la tapa de la artesa. El resto del hielo de la formulación se agregó poco a poco con el fin de mantener la temperatura de la masa entre 2 y $6^{\circ} \mathrm{C}$ para evitar la desnaturalización de la proteína. Luego del picado, la emulsión obtenida fue embutida en una tripa artificial, calibre $26 \mathrm{~mm}$, con una embutidora marca Premier Super Grinder. Las salchichas se porcionaron en unidades de $\pm 16 \mathrm{~cm}$ de longitud, luego de lo cual se llevaron a cocción en agua caliente a $80^{\circ} \mathrm{C}$ hasta alcanzar una temperatura en el centro térmico de $72^{\circ} \mathrm{C}$, medidos con termómetro digital de punzón Brixco modelo Waterproof. Una vez alcanzada la temperatura de $72^{\circ} \mathrm{C}$ se retiraron del agua caliente y se sometieron a enfriamiento con agua fría hasta alcanzar una temperatura interna de $30^{\circ} \mathrm{C}$, procediendo a su almacenamiento en refrigeración a $4^{\circ} \mathrm{C}$ hasta el momento del análisis. 


\section{Formulación de las salchichas}

Se realizó una serie de pruebas para determinar la proporción de los ingredientes para la formulación de las salchichas, con el fin de obtener un producto de apariencia y sabor semejantes a las salchichas tradicionales. En la Tabla 1 se detalla la formulación ensayada:

Tabla 1 Formulación de la salchicha

\begin{tabular}{lr}
\hline \multicolumn{1}{c}{ INGREDIENTES } & \multicolumn{1}{c}{$\%$} \\
\hline Carne roja de la cabeza del atún & 43,88 \\
Grasa de cerdo & 10,45 \\
hielo & 11,28 \\
Almidón de yuca & 6,22 \\
Proteína de soya & 25,12 \\
Sal & 2,00 \\
Azúcar & 0,14 \\
Dióxido de titanio & 0,25 \\
Óxido de hierro & 0,047 \\
Polifosfatos & 0,156 \\
Nitrito de sodio & 0,057 \\
Ácido ascórbico & 0,05 \\
Pimienta & 0,14 \\
Ajo & 0,15 \\
Humo líquido & 0,03 \\
Condimento para salchicha & 0,026 \\
\hline
\end{tabular}

\section{Análisis de las salchichas}

La determinación de humedad, proteína, grasa, carbohidratos y cenizas se realizó por triplicado según métodos de la Asociación Oficial de Química Analítica (AOAC2003).

\section{Análisis microbiológico}

Se analizaron seis salchichas a las 24 horas de elaboradas. Se realizó por ducplicado el recuento de aerobios mesófilos, coliformes totales (CT) y fecales (CF), Salmonella y Staphylococcusaureus. Se siguió la metodología señalada por las Normas Técnicas Colombianas (NTC 4779 y 4458).

\section{Evaluación sensorial}

La acogida de las salchichas se evaluó con base en el olor, color, sabor y textura, utilizando una escala hedónica de 4 puntos, con los siguientes descriptores: me desagrada mucho $=1$, me desagrada un $\operatorname{poco}=2$, me gusta mucho=3 y me gusta muchísimo $=4$.
Las muestras fueron calentadas en agua a una temperatura de $70^{\circ} \mathrm{C}$, cortadas en trozos de $1,5 \mathrm{~cm}$ e identificadas con números aleatorios de tres cifras. La evaluación fue hecha en un área ventilada, con buena iluminación, libre de olores extraños y con un panel de 20 evaluadores no entrenados, a los cuales se les suministró una ficha de evaluación.

\section{Análisis del perfil de textura (TPA)}

Se utilizó un texturómetro Marca Shimazu® y se determinó el perfil de textura por medio del Rheometer software. Para esto se cortaron rodajas de salchicha de $1,5 \mathrm{~cm}$ de grosor aproximado, y se dejaron reposar por una hora a temperatura ambiente dentro de una bolsa de polietileno, para evitar la pérdida de humedad (Isaza et al., 2010). El método de TPA fue realizado mediante la aplicación de una fuerza de compresión dos veces sucesivas en las muestras, con el fin de simular la masticación humana, donde fue obtenida la curva fuerza/tiempo y calculados los siguientes parámetros: cohesividad, dureza, adhesividad, fracturabilidad, gomosidad, masticabilidad y elasticidad. En la prueba de corte, las muestras fueron colocadas sobre la mordaza (Isaza et al., 2010).

\section{Análisis estadístico}

Para determinar diferencias estadísticas, se utilizó el programa Minitab-16 (StatisticalSoftware, USA 2011).

\section{Resultados y discusión}

\section{Análisis proximal}

De acuerdo a la Tabla 2, el nivel de grasa encontrado en las salchichas es más bajo que el reportado para salchichas elaboradas con carne de res, carnero, pollo y cerdo en las que se alcanzan valores que oscilan entre un 24 y $45 \%$ (García et al., 2005). También es más bajo que el nivel de grasa para salchichas elaboradas a partir de tilapia roja, en las cuales se hallan valores promedios de 14,68+/0,41 (Hleap2010). Así mismo, es menor a los límites máximos establecidos por las normas colombianas para el contenido en grasa de salchichas elaboradas con carne de bovino $(21 \%)$ y de porcino, o mezcla de bovino y porcino (35\%).

Tabla 2. Análisis proximal de la salchicha de atún.

\begin{tabular}{ccccc}
\hline $\begin{array}{c}\text { Humedad } \\
(\%)\end{array}$ & $\begin{array}{c}\text { Grasa } \\
(\%)\end{array}$ & $\begin{array}{c}\text { Proteínas } \\
(\%)\end{array}$ & $\begin{array}{c}\text { Cenizas } \\
(\%)\end{array}$ & $\begin{array}{c}\text { Carbo- } \\
\text { hidratos } \\
(\%)\end{array}$ \\
\hline 67,25 & 8,2 & 17,3 & 1,95 & 5,3 \\
\hline
\end{tabular}


El contenido de humedad, y carbohidratos fue mayor que los reportados para salchichas de tilapia roja $(65,8$ y 3,41) (Hleap 2010). Es probable que estos valores elevados en el contenido de humedad se deban a que el atún, el almidón de yuca y la proteína de soya utilizado se caracterizan por una elevada retención de agua (García et al., 2005). Sin embargo, estos valores fueron inferiores a los reportados por Cabello et al. (1995) (73,7\%) en salchichas de pescado a base de fauna acompañante de camarón. El contenido de proteínas fue superior a los establecidos por la norma colombiana para salchichas de cerdo y bovino NTC 1325 (11 y $12 \%$ respectivamente), a los reportados para salchichas de pescado 12,2\% (Cabello et al., 1995; Tinedo 1998) y a los hallados por García et al., (2005) en formulaciones de salchichas con atún y carne de res. Mientras que el contenido de cenizas fue menor en comparación con el embutido de tilapia (1,95\%).

\section{Análisis microbiológicos}

Los resultados de estos análisis son indicativos de buena calidad sanitaria. La Tabla 3 muestra los valores obtenidos para el recuento de aerobios mesófilos, coliformes totales y fecales, y Salmonella. Todos los recuentos de microorganismos se encontraron por debajo de los límites establecidos en las normas colombianas INVIMA y NTC 4779 y 4458 . Ellas establecen valores de aerobios mesófilosen $1.0 \times 10^{5} \mathrm{UFC} / g$, coliformes fecales < 3. Staphylococcusaureus salmonella en $25 \mathrm{~g}$ ausente. La carne de atún utilizada como materia prima para la elaboración de las salchichas es altamente susceptible de contaminación, y proveen un medio favorable para el crecimiento de microorganismos que pueden contaminar el tejido. Sin embargo, el bajo conteo microbiológico puede ser explicado por el uso de materia prima fresca y buen manejo sanitario, alta temperatura en la cocción, rápido enfriamiento del producto de hasta $-8^{\circ} \mathrm{C}$, y al uso de envolturas impermeables. Estos resultados coinciden con los reportados por Hing et al. (1995).

Tabla 3. Análisis microbiológicos de la salchicha de atún

\begin{tabular}{l|c}
\hline Recuento de mesófilos aeróbicos & $6 \times 10^{1} \mathrm{col} / \mathrm{g}$ \\
Recuento de mesófilos anaeróbicos & 0 \\
Recuento de coliformes totales & 0 \\
Recuento de coliformes fecales & 0 \\
Salmonella en 25g & Negativo \\
Staphylococcusaureus & Negativo \\
\hline
\end{tabular}

\section{Evaluación sensorial}

Los resultados obtenidos para la evaluación sensorial de las salchichas de atún, en función de las caracterís- ticas, fue 3,6 para el sabor, 3,6 para el olor, 3,7 para el color y 3,5 para la textura.

La salchicha alcanzó valores superiores en todos los atributos, encontrándose entre "me gusta muchísimo" y "me gusta mucho". Los valores más bajos asignados por los evaluadores fueron atribuidos a la textura debida a la cantidad de proteínas en la formulación. El color fue similar al de las salchichas comerciales.

De acuerdo con los resultados obtenidos por parte de los panelistas, las salchichas presentaron una textura suave que fue de su agrado. Resultados contrarios fueron señalados por Park et al (1988). Ellos evaluaron salchichas elaboradas con merluza en reemplazo parcial de la carne de res. Encontraron que los panelistas rechazaban la textura suave, afirmando que esta característica representa uno de los mayores problemas cuando se adicionan pulpas de productos marinos en el proceso de elaboración de las salchichas.

\section{Análisis del perfil de textura (TPA)}

Los resultados de TPA se muestran en la Tabla 3. Al evaluar la textura de la salchicha de atún, se observó una dureza inferior a la salchicha de carne de res reportados por Herrero et al. 2008, una mayor elasticidad, mayor cohesividad y menor gomosidad. Los valores de adhesividad obtenidos son negativos. Ello indica que la textura de la salchicha es pegajosa o adhesiva. Cuando el producto es consumido éste se adhiere al paladar, lo que conlleva a realizar un trabajo adicional para retirarlo. Disminuyó su masticabilidad, es decir, requiere menos esfuerzo para masticarla.

Tabla 4. Análisis de textura de la salchicha de atún

\begin{tabular}{lcc}
\hline Parámetro & $\begin{array}{c}\text { Valor promedio } \\
\text { por repeticiones }\end{array}$ & $\begin{array}{c}\text { Desviación } \\
\text { estándar } \pm\end{array}$ \\
\hline Dureza & 25,6 & 0,3 \\
Elasticidad & 93.3 & 4,3 \\
Cohesividad & 78,3 & 5,2 \\
Gomosidad & $-0,18$ & 0,004 \\
Masticabilidad & 15,8 & 0,45 \\
\hline
\end{tabular}

\section{Conclusiones}

A partir de la carne roja de atún, se obtuvo una salchicha con un alto contenido de proteína y bajo contenido de grasa y minerales, con buenas características microbiológicas y sensoriales. 


\section{Referencias}

AOAC. Association of official methods of analytical chemist.2003. Washington, U.S.A., Chapter 32: 1, 2, 5 y 14 ()

Cabello A et al. 1995 Nuevos productos pesqueros en la dieta del venezolano. INIA Divulga 49(12): 19-23.

García M, Izquierdo P, Faria J. 2005. Formulación de salchichas con atún y carne: vida útil y aceptabilidad. Revista científica FCV-LUZ 15(3): 272-278.

Granados C. et al. 2012Elaboración de chorizo a base de pescado. Vitae 19 (Supl. 1), S237-S239

Herrero AM, de la Hoz L, Ordóñez JA, Herranz B, Romero MD, Cambero MI. Tensile properties of cooked meat sausages and their correlation with texture profile analysis (TPA) parameters and physico-chemical characteristics, Meat Science 2008;80(3):690-696

Hing FS, Yu-ang N, Cavaletto CG. Stability of fish sausage at low temperature storage J. food Sci. 1996; 37(2): 191 194.

Hleap J, Velasco V. Analysis of the properties of texture during the storage of sausage made from red tilapia (Oreochromis sp.). Rev Bio Agro. 2010; 8(2): 46-56.

Hleap J, Molina A, 2008. Proceso de elaboración de salchichas a partir de tilapia roja (Oreochromissp) con adición de almidón de sagú (Maranthaarundinacea). Manual de Transferencia Tecnológica. Universidad Nacional de Colombia. Palmira, 57 páginas.
Isaza JM, Londoño LM, Restrepo DA, Cortes M, Suárez H. Producción y propiedades funcionales de plasma bovino hidratado en embutido tipo salchichón. Rev Colom Cien Pecu 2010; 23(2):199-206.

Izquierdo P, García A, Allara M, Rojas E, Torres G, González P. Análisis proximal, microbiológico y evaluación sensorial de salchichas elaboradas a base de Cachama Negra (Colossomamacropomum). Revista Científica 2007; 17(3): 294-300.

Noguez J, Dreyfus M, Robles H. Análisis de la pesca del atún con palangre en el golfo de México durante las fases de luna Ilena. Revista Hidrobiológica. 2007; 17(2): 91-99.

Norma Técnica Colombiana. NTC 4458, 2007, Método para el recuento de coliformes y Escherichiacoli.

Norma Técnica Colombiana. NTC 4779, 2007, Microbiología de alimentos. Método horizontal para el recuento de estafilococos coagulasa positiva (staphylococcusaureus y otras especies).

Norma Técnica Colombiana. NTC. 1325 Productos cárnicos no enlatados. (2008)

Park E, Brekke C), Branen A, Use of pacific hake(Merlucciusproductus) in a frankfurter formulation, J Food Sci 1988; 44: 1637-1645.

Tinedo $V, 1998$. Alternativas en la elaboración de embutidos a base de pulpa de pescado. Universidad Central de Venezuela. Instituto en Ciencia y Tecnología de Alimentos. 24-48pp. 